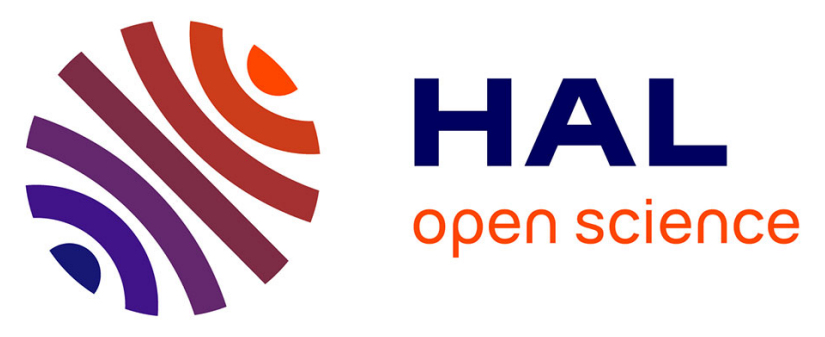

\title{
Mushroom Body Efferent Neurons Responsible For Aversive Olfactory Memory Retrieval In Drosophila
} Julien Séjourné, Pierre-Yves Plaçais, Yoshinori Aso, Igor Siwanowicz, Séverine

Trannoy, Vladimiros Thoma, Stevanus Rio Tedjakumala, Gerald M. Rubin, Paul Tchénio, Kei Ito, et al.

\section{To cite this version:}

Julien Séjourné, Pierre-Yves Plaçais, Yoshinori Aso, Igor Siwanowicz, Séverine Trannoy, et al.. Mushroom Body Efferent Neurons Responsible For Aversive Olfactory Memory Retrieval In Drosophila. Nature Neuroscience, 2011, 10.1038/nn.2846 . hal-00653182

\section{HAL Id: hal-00653182 https://hal.science/hal-00653182}

Submitted on 19 Dec 2011

HAL is a multi-disciplinary open access archive for the deposit and dissemination of scientific research documents, whether they are published or not. The documents may come from teaching and research institutions in France or abroad, or from public or private research centers.
L'archive ouverte pluridisciplinaire HAL, est destinée au dépôt et à la diffusion de documents scientifiques de niveau recherche, publiés ou non, émanant des établissements d'enseignement et de recherche français ou étrangers, des laboratoires publics ou privés. 


\section{Mushroom Body Efferent Neurons Responsible For Aversive Olfactory Memory Retrieval In Drosophila}

Julien Séjourné ${ }^{1}$, Pierre-Yves Plaçais ${ }^{1}$, Yoshinori Aso ${ }^{2,3}$, Igor Siwanowicz ${ }^{2}$, Séverine Trannoy $^{1}$, Vladimiros Thoma ${ }^{2}$, Stevanus R. Tedjakumala ${ }^{2}$, Gerald M. Rubin ${ }^{3}$, Paul Tchénio ${ }^{1,4}$, Kei Ito ${ }^{5}$, Guillaume Isabel ${ }^{1}$, Hiromu Tanimoto ${ }^{2,6}$ and Thomas Preat ${ }^{1,6}$

${ }^{1}$ Genes and Dynamics of Memory Systems, Neurobiology Unit, CNRS, ESPCI, 75005 Paris, France.

${ }^{2}$ Max-Planck-Institut für Neurobiologie, Am Klopferspitz 18, D-82152 Martinsried, Germany.

${ }^{3}$ Janelia Farm Research Campus, Howard Hughes Medical Institute, Ashburn, Virginia 20147, USA.

${ }^{4}$ Nanooptique et Physiologie Intégrée, Université Paris-Sud, CNRS UPR 3321, Batiment 505, Campus d'Orsay, 91400 Orsay, France.

${ }^{5}$ Institute of Molecular and Cellular Biosciences, The University of Tokyo, Bunkyo-ku, Tokyo 113-0032, Japan.

${ }^{6}$ These authors contributed equally to this work. 
Aversive olfactory memory is formed in the Drosophila mushroom bodies (MB). Memory retrieval requires MB output, but it remains unknown how a memory trace in the MB drives conditioned avoidance of a learned odour. To identify neurons involved in olfactory memory retrieval, we performed an anatomical and functional screen of defined sets of MB extrinsic neurons. Here we show that MB-V2 neurons are essential for retrieval of both short- and long-lasting memory, but neither for memory formation nor for memory consolidation. We further show that MB-V2 are cholinergic efferent neurons that project from the MB vertical lobes to the middle superiormedial protocerebrum and the lateral horn (LH). Notably, the odour response of MB-V2 neurons is modified after conditioning. As the $\mathrm{LH}$ is implicated in innate responses to repellent odorants, we propose that during memory retrieval, MB-V2 neurons reinforce the olfactory pathway involved in innate odour avoidance.

Different odours induce innate approach or avoidance behaviours in Drosophila. Innate odour responses can be modulated by experience, such as associative learning. After simultaneous exposure to an electric shock and an odorant, flies form aversive memory and show robust conditioned odour avoidance that lasts for hours to days, depending on the training protocol ${ }^{1-3}$. The neural pathways for odour or shock processing and signal integration in the fly brain have been intensely studied in recent years. Odour information is first represented in the antennal lobes in the form of olfactory receptor neuron activity ${ }^{4}$. Projection neurons then convey this information to higher order processing centres ${ }^{4}$ : the mushroom bodies (MB) and the lateral horn (LH). In contrast, aversive reinforcement signals in response to electric shock are relayed to the $\mathrm{MB}$ via dopaminergic neurons ${ }^{5-7}$. The olfactory and 
electric shock signals are integrated in the MB to form aversive olfactory memory ${ }^{1,2}$. The MB are however dispensable for innate avoidance of the repellent odours ${ }^{8,9}$.

In adult Drosophila, the MB consist of approximately 2000 Kenyon cells per brain hemisphere, which may be classified into three major types based on their axonal projection: $\gamma$ neurons, which form only a medial lobe, $\alpha / \beta$ neurons, whose axons branch to form a vertical $(\alpha)$ and a medial $(\beta)$ lobe, and $\alpha^{\prime} / \beta^{\prime}$ neurons, which also form a vertical $\left(\alpha^{\prime}\right)$ and a medial ( $\left.\beta^{\prime}\right)$ lobe ${ }^{10}$. Functional brain imaging has revealed localised activation of cAMP/PKA signalling in the $\mathrm{MB} \alpha$ lobe in response to simultaneous cholinergic and dopaminergic stimulation $^{11,12}$, that represent respectively the odorant and electric shock pathways. Following associative conditioning, calcium imaging studies have shown that a short-term memory trace is formed in the $\alpha^{\prime} / \beta^{\prime}$ neurons ${ }^{13}$, and a long-term one in $\alpha$ lobes $^{14}$. Previous studies have shown that the output of the $\alpha / \beta$ neurons is necessary for the retrieval of all phases of olfactory memory ${ }^{15,16}$, but the neural circuits that translate the associative memory trace in the MB into conditioned odour avoidance remain unknown.

To understand the neuronal basis of memory retrieval, we conducted a functional screen based on collections of GAL4 driver lines for MB-extrinsic neurons ${ }^{17,18}$ (data not shown). We blocked the neurotransmission of GAL4-expressing cells during the retrieval of different forms of short- and long-lasting memories using $\mathrm{Sh}^{\text {ts }}{ }^{[19]}$, a dominant negative temperature sensitive variant of Dynamin that can reversibly block synaptic vesicle endocytosis at elevated temperatures. Because neurons responsible for memory retrieval should receive synaptic inputs from the $\mathrm{MB}$ and project to other parts of the brain, we characterized the polarity of $\mathrm{MB}$ extrinsic neurons by expressing a presynaptic marker ${ }^{20}$. We further characterized the identified $\mathrm{MB}$ output neurons with regard to neurotransmitter and physiological response properties using immunohistochemistry and functional calcium imaging. 


\section{Results}

\section{MB-V2 neurons are efferent to the MB}

In a preliminary screen, we functionally analyzed 58 enhancer-trap GAL4 lines showing expression in putative $\mathrm{MB}$ extrinsic neurons for a defect in aversive memory retrieval (data not shown). We identified a particular set of MB extrinsic neurons, the MB-V2, as a candidate for the memory retrieval pathway. MB-V2 neurons are labelled in the GAL4 driver lines $N P 2492^{18}$ (Fig. 1a-c and Supplementary Fig. 1a) and $M Z 160^{17,18}$ (Supplementary Fig. 1d). The MB-V2 neurons have their cell bodies posterior to the LH (Supplementary Fig. 1b,e), and arborise in the vertical lobes of the $\mathrm{MB}$, the lateral part of the middle superiormedial protocerebrum (msmpr) just posterior to the vertical lobes, and the LH (Fig. 1a-c). Whereas membrane-targeted markers (mCD8::GFP or myr::RFP) uniformly label MB-V2 processes in all three target neuropils (Fig. 1a-c and Supplementary Fig. 2), the signals of the presynaptic marker Syt::GFP (a fusion of eGFP and the synaptic vesicle protein Synaptotagmin ${ }^{20}$ ) were highly enriched in the msmpr and LH but not detectable in the MB vertical lobes (Fig. 1d-f). We observed similar results with other presynaptic markers (Syt::HA ${ }^{21}$ and nSyb::GFP ${ }^{17}$ ) (Fig. 1m-r and Supplementary Fig. 2d-i). Quantification of Syt::HA and mCD8::GFP signal intensity in the MB-V2 terminals confirmed that Syt::HA is highly enriched in the msmpr and the LH, but not on MB (Supplementary Fig. 2j). Thus, MB-V2 neurons are likely to relay information from the MB vertical lobes to the msmpr and LH.

Flp-out clones ${ }^{22}$ of single MB-V2 neurons revealed two stereotyped neuron classes: MB-V2 $\alpha$ and MB-V2 $\alpha$ '. MB-V2 $\alpha$ neurons innervate across the shaft of the $\alpha$ lobe of the ipsilateral $\mathrm{MB}$ and terminates in the msmpr and dorsal area of the $\mathrm{LH}$ of both hemispheres 
(Fig. 1g-i), whereas MB-V2 $\alpha$ ' neurons arborise in the tip of the $\alpha$ ' lobe of the ipsilateral MB and project to the msmpr and the ventral area of the LH (Fig. 1j-l) of both sides. The collaterals of MB-V2 $\alpha^{\prime}$ also terminate in a brain area ventral medial to the LH. MB-V2 $\alpha$ and MB-V2 $\alpha^{\prime}$ neurons are labelled in NP2492, whereas only the MB-V2 $\alpha$ appears to be labelled in MZ160 (Supplementary Videos 1 and 2). Neurons in the MB-V2 cluster are the only GAL4-expressing cells that are clearly shared by NP2492 and MZ160.

\section{MB-V2 output is required for olfactory memory retrieval}

To address the role of the MB-V2 neurons in innate odour responses, we first measured avoidance of 4-methylcyclohexanol (MCH) and 3-octanol (OCT), while blocking the output of MB-V2 neurons. Consistent with previous reports showing that MB are dispensable for innate odorant avoidance ${ }^{8,9}$, we found that the odour avoidance of both NP2492/UAS-shits and MZ160/UAS-shits flies at restrictive temperature was normal (Table 1). We then examined the effect of the transient blockade of these neurons during the test on 2-h memory retrieval after a single cycle conditioning. We observed a strong memory impairment in both $N P 2492 / U A S-s h i^{t s}$ and $M Z 160 / U A S-s h i^{t s}$ flies (Fig. 2a).

To assess whether MB-V2 output is also required for memory formation, we next blocked these neurons transiently during training and consolidation, and tested the memory 2 hours later at the permissive temperature. Interestingly, blocking the MB-V2 during training and consolidation did not significantly impair 2-h memory performance (Fig. 2b). Moreover, no memory defect was observed when flies were conditioned and tested at permissive temperature (Fig. 2c).

Memory directly after training was also reduced significantly when MB-V2 output was blocked continuously (Supplementary Fig. 3a). As MB-V2 output is not required for 
memory acquisition (Fig. 2a), this result suggests that immediate-memory retrieval also depends on MB-V2 output.

Output of $\alpha / \beta$ neurons is required for appetitive memory retrieval ${ }^{23}$. In order to determine whether MB-V2 output is required for all types of memory retrieval, we performed appetitive conditioning, where electric shocks are replaced by sugar reward ${ }^{24}$. A sugar response defect was observed in NP2492/UAS-shits flies tested at restrictive temperature (Supplementary Fig. $3 \mathbf{b}_{1}$ ) that may correspond to a motivation defect, and therefore appetitive memory could not be assessed in these flies. No sugar defect was observed in $M Z 160 / U A S-s h i^{t s}$ flies at restrictive temperature (Supplementary Fig. $3 \mathbf{b}_{2}$ ) and no appetitive memory defect was detected in these flies when MB-V2 neurons were blocked during retrieval (Supplementary Fig. 3c). These results show that MB-V2 $\alpha$ output is dispensable for appetitive memory retrieval.

The odorants are highly repellent to naïve flies at the concentration we typically use for conditioning (e.g. Table 1). To address whether the requirement of MB-V2 for memory retrieval depends on how aversive the odour is, we next used odorants that are neutral to naïve flies for conditioning. We found that $\mathrm{OCT}$ and $\mathrm{MCH}$ are no longer aversive when diluted 300 times compared to the usual concentration $\left(1.2 \times 10^{-6} \mathrm{M}\right.$ and $1.08 \times 10^{-6} \mathrm{M}$ respectively, data not shown). When conditioning and test were performed with the diluted odours, both NP2492/UAS-shits and MZ160/UAS-shits flies showed a robust 2-h memory impairment (Supplementary Fig. 2d), suggesting that MB-V2 output is required for aversive memory retrieval irrespective of the repulsiveness of odorants.

\section{GAL80 expression rescues the memory retrieval impairment}


Neurons in the MB-V2 cluster are the only GAL4-expressing cells that are clearly shared by $N P 2492$ and $M Z 160$, but each line shows expression in additional brain regions, such as the fan-shaped body in NP2492 (Supplementary Fig. 1a and Supplementary Video 1) and the optic lobes in MZ160 (Supplementary Fig. 1c,g and Supplementary Video 2). Even though the behavioural phenotype is common and very specific for the retrieval of aversive memory (Fig. 2 and Supplementary Fig. 3), one could not formally exclude possibilities that different sets of neurons outside MB-V2 happen to induce the same memory phenotype. To confirm that blocking MB-V2 neurons is the cause of the memory impairment, we aimed to use the intersectional approach and searched for GAL80 lines that can preferentially silence GAL4 activity in MB-V2. $C h a^{3.3 k b}-G A L 80^{\text {25] }}$ reduced the reporter expression in MB-V2 in NP2492 and MZ160 (Fig. 3a-b, Supplementary Fig. 1a,c,d,f and Supplementary Video 3). To improve the specificity of the intersectional manipulation to MB-V2, we also replaced the GAL4 gene in NP2492-GAL4 with GAL80 ${ }^{26}$ (see Methods section for details). The resulting line, NP2492-GAL80, appeared to largely recapitulate the expression pattern of NP2492-GAL4, as it silenced the majority of reporter expression in NP2492-GAL4 (Supplementary Fig. 1h). In combination with MZ160, NP2492-GAL80 significantly reduced the reporter expression in MB-V2 (Fig. 3c, Supplementary Fig. 1i,j and Video 4).

Consistent with the anatomical data, impaired 2-h memory of $M Z 160 / U A S-s h i^{\text {ts }}$ flies was fully rescued by combination to $C h a^{3.3 k b}$-GAL80 (Fig. 3d) and NP2492-GAL80 (Fig. 3e). As both $C h a^{3.3 k b}$-GAL80 and NP2492-GAL80 suppress most of the GAL4-expressing cells in NP2492 driver (Supplementary Fig. 1c,h), no rescue experiments were performed with these genotypes. These results strongly suggest that the memory retrieval defect observed in $M Z 160 / U A S-s h i^{t s}$ flies is due to MB-V2 blockade. 


\section{MB-V2 output is required to retrieve consolidated memories}

Aversive odour memory in Drosophila consists of various phases ${ }^{1,3}$. Short-term memory (STM) can be formed by a single cycle of training and is labile. Several cycles of massed or spaced training (i.e. without or with rest intervals between conditioning cycles, respectively) induce distinct forms of long-lasting memory ${ }^{3}$ : anaesthesia-resistant memory (ARM), and long-term memory (LTM), respectively ${ }^{15}$.

Memory 24 hours after both massed and spaced conditioning was strongly impaired in both genotypes when MB-V2 output was blocked during testing (Fig. 4a,b). Consistent with the fact that MB-V2 output is not required during training or consolidation after a single conditioning cycle (Fig. 2b), blockade of MB-V2 output during spaced training and the following two hours does not affect 24-h memory (Fig. 4c). Taken together, these results demonstrate that the retrieval of all phases of aversive olfactory memory, i.e. STM, LTM, and $\mathrm{ARM}$, requires the MB-V2 output whereas training and consolidation do not.

\section{MB-V2 neurons are cholinergic}

To characterize MB-V2 with regard to neurotransmitter, we examined colocalization of MB-V2 and markers for various transmitters. Their cell bodies were not colocalized with Drosophila Vesicular Glutamate Transporter, Tyrosine Hydroxylase, Glutamate Decarboxylase 1 (DVGLUT, TH, GAD1 resp., Fig. 5a-c), as well as octopamine/tyramine or serotonin (data not shown). We confirmed the results obtained with NP2492 with another

driver line, $R 71 D 08$ (Supplementary Fig. 4), constructed as described in reference ${ }^{[27]}$ (see methods). The R71D08 driver strongly labels a smaller number of MB-V2 cluster neurons (Supplementary Fig. 1k and Supplementary Video 5). Consistently with the behavioural 
results obtained with $N P 2492$ and MZ160 drivers, we observed a 2-h memory impairment when MB-V2 output is blocked during the test using the R71D08 driver (Supplementary Fig. 5a). Moreover, no memory defect was observed when $R 71 D 08 / U A S-s h i^{t s}$ flies were conditioned and tested at permissive temperature (Supplementary Fig. 5b) and the odour avoidance of R71D08/UAS-Shis flies was normal at restrictive temperature (Supplementary Fig. 5c).

We next examined Choline Acetyltransferase (ChAT)-immunoreactivity in the presynaptic terminals of MB-V2, as the antibody does not significantly labels cell bodies ${ }^{28}$. We found that a fraction, but not all, of the terminals of MB-V2 neurons colocalized with ChAT (Fig. 5d-i and Supplementary Fig. 6 and 7), indicating that at least some of the MBV2 neurons are cholinergic. We also confirmed the colocalization with ChAT with the terminals of single MB-V2 neurons (Fig. 5j-o). The presynaptic terminals of MB-V2 were not immunoreactive to DVGLUT or GAD1 (Supplementary Fig. 8 and 9), consistently with the observations of the cell bodies (Fig. 5a-c and Supplementary Fig. 4). The conclusion that MB-V2 are cholinergic is in line with the suppression of transgene expression in MB-V2 by Cha ${ }^{3.3 k b}-G A L 80$ (Fig. 3b).

\section{MB-V2 neurons show a reduced response to trained odorants}

To understand the physiological role of the MB-V2 neurons during aversive olfactory memory retrieval, we analyzed the MB-V2 response to odours using in vivo calcium imaging. We expressed GCaMP 3.0, a calcium-sensitive fluorescent protein ${ }^{29}$ using NP2492 driver and recorded the fluorescence of GCaMP3 from the MB-V2 neurons in the region of the MB vertical lobes and msmpr in naïve flies (Fig. 6a). A strong calcium increase was detected in response to benzaldehyde, 3-octanol, methylcyclohexanol and isoamylacetate (Fig. 6c-f) 
compared to that obtained with pure paraffin oil (Fig. $6 \mathbf{b}$ and $\mathbf{h})$. Responses to all odours were similar in MB-V2 neurons (Fig. 6h) and decreased rapidly after the end of the stimulation (OCT, MCH: Fig. 6g; isoamylacetate and benzaldehyde, data not shown). These results show that the MB-V2 neurons respond to odours by increasing intracellular calcium concentration.

As the MB-V2 neurons respond to odorants, we asked whether the MB-V2 show training-induced change of their response properties to the conditioned stimulus after aversive training. NP2492/+; UAS-GCaMP3/+ flies showed a wild-type memory (Fig. 7a) suggesting that expression of the GCaMP3 reporter in the MB-V2 neurons does not affect their physiology. Three hours after the flies had been trained with OCT as the conditioned stimulus (paired training, Fig. 7b), the calcium response to OCT in MB-V2 neurons was significantly decreased compared to $\mathrm{MCH}$ response (Fig. 7c,g). This decrease is the result of the temporal pairing of the odorant and the shock stimuli, as no such decrease was observed (Fig. 7e,i) when OCT and electric shocks were delivered 120 seconds apart (unpaired training, Fig. 7b). Consistently, the relative response of OCT to $\mathrm{MCH}$ in paired flies was reduced by $45 \%$ compared to unpaired flies (Fig. 7k). Similar results were obtained when $\mathrm{MCH}$ was used as the conditioned odorant (Fig. $\mathbf{7 d}, \mathbf{f}, \mathbf{h}, \mathbf{j}, \mathbf{l})$. Thus, the MB-V2 neurons transmit a memory trace in the MB by decreasing their calcium response to the trained odorant.

\section{DISCUSSION}

Here, we described for the first time the function of a class of MB efferent neurons in Drosophila, MB-V2. We found two distinct populations of MB-V2 neurons, MB-V2 $\alpha$ and MB-V2 $\alpha^{\prime}$ that innervate the shaft of the MB $\alpha$ lobe and the tip of the MB $\alpha^{\prime}$ lobe, respectively. The MB $\alpha$ lobe has been implicated as a site of olfactory learning that employs an associative synergy of cholinergic and dopaminergic inputs to activate cAMP/PKA 
signalling $^{11,12}$. In addition to the MB, both MB-V2 $\alpha$ and MB-V2 $\alpha^{\prime}$ arborise in the neuropil just posterior to the MB vertical lobes and in the lateral horn (LH), the other major secondary olfactory centre. By transiently blocking synaptic transmission, we found that MB-V2 output is required specifically to retrieve aversive olfactory memory.

Although the molecular pathways underlying STM, LTM, and ARM differ ${ }^{1,2,15}$, our results suggest that a common neuronal network is used to translate the memory trace in the Kenyon cells projecting to the MB vertical lobes into avoidance behaviour. Other MB output neurons may also be involved in short-lasting memory retrieval, as blockade of MB-V2 output, while fully abolishing consolidated memories, only partially impairs short-lasting memories. Interestingly, MB-V2 neurons are not required for the retrieval of sugar-associated memory and thus other neurons must provide the output for appetitive memory. Finally, no significant behavioural differences were observed with the aversive protocol when both V2 $\alpha$ and $\alpha^{\prime}(N P 2492)$ or only $\mathrm{V} 2 \alpha(M Z 160)$ were blocked, and the role of MB-V2 $\alpha^{\prime}$ neurons remains to be elucidated.

Functional optical imaging revealed that MB-V2 neurons respond to odorants and decrease their response to the trained odorant. Thus, aversive olfactory conditioning represses the activity of MB-V2 cholinergic neurons. How can an increased calcium response in the $\mathrm{MB}$ lobes to the learned odour $(\mathrm{CS}+)^{13,14}$ induce a decreased calcium response in the MB-V2 neurons? Inhibitory neurons that project onto MB-V2 may be recruited during conditioning. Strikingly, the PE1 neuron in Apis mellifera relays information from the MB to the LH and shows a reduced response to $\mathrm{CS}+$ after appetitive training ${ }^{30}$. It has been proposed that this reduced response could be due to inhibitory GABAergic inputs on $P E 1^{31}$. The conservation between bees and flies suggest a particularly important role of this circuit in both insects. Alternatively, the decreased calcium response of MB-V2 to the CS+ may correspond to synaptic depression. As shown in mammals, long-term potentiation or depression may recruit 
differential molecular mechanisms, such as NMDA receptor activation ${ }^{32,33}$ and retrograde signalling by endocannabinoids ${ }^{32-34}$. Such depression may occur in the MB-efferent synapses during memory acquisition ${ }^{35}$, although underlying mechanisms are still yet to be identified in Drosophila. Interestingly, in Drosophila, the GABAergic APL neuron that projects onto the MB shows a reduced response to the trained odour ${ }^{36}$. Therefore, the plasticity mechanism that occurs at the MB synapse could be shared between APL and MB-V2 neurons.

How is the reduced calcium response to the conditioned odorant in MB-V2 neurons translated into the avoidance behaviour? We found that MB-V2 project to the $\mathrm{LH}$, a brain region that receives direct olfactory inputs and is involved in the innate avoidance of repellent odorants $^{8,9}$. Thus, in Drosophila, two olfactory pathways converge toward the LH: one innate odorant avoidance pathway that goes directly from antennal lobes to the LH, and an associative learning-dependant one that requires integration of the negative value of shock by the MB. We found the pre-synaptic cholinergic terminals of MB-V2 to be in close proximity to GABAergic neurons in the LH (Supplementary Fig. 9). Thus, reduced calcium response in MB-V2 neurons upon exposure to the conditioned odorant could lead to an enhanced avoidance to the aversive odour by reducing GABAergic inhibitory input on projection or LH neurons. Alternatively, it has been shown in mammals that activation of muscarinic receptors by acetylcholine can directly induce $\mathrm{LTD}^{37}$. Thus, the reduced calcium response observed to $\mathrm{CS}+$ in MB-V2 could directly result in lower inhibition of olfactory signalling mediated by the LH.

MB-V2 neurons also have pre-synaptic terminals in the msmpr area. As there are interneurons that connect the dorsal LH and $\mathrm{msmpr}^{38}$, the learned odor may induce avoidance behavior at the msmpr upon the stimulation of the LH interneurons via MB-V2. The functional relationships between the $\mathrm{LH}$ and the msmpr, both targets of the MB-V2 neurons, remains to be resolved. 
Note: Supplementary information is available on the Nature Neuroscience website

ACKNOWLEDGMENTS We thank Joachim Urban and Gehrard M. Technau for the MZ160 line and the members of the NP consortium for the NP2492 line. We thank Jean-Maurice Dura for the $y^{1} w^{1118} ; G A L 80\left[y^{+}\right], S b$ line and for helpful discussions. We thank Loren L. Looger for the UAS-GCaMP3 line. We thank T. Kitamoto for the GAD1-GAL80 line. We thank Vincent Hakim, Isabelle Rivals and members of our laboratories for helpful discussions. This work was supported by grants from the Japan Society for the Promotion of Science (K.I.), the Agence Nationale pour la Recherche (T.P.), the Fondation Bettencourt-Schueler (T.P.), the Fondation pour la Recherche Médicale (T.P.), the Emmy-Noether Program from Deutsche Forschungsgemeinschaft (H.T.), the Bernstein focus Learning from BMBF (H.T.) and the Max-Planck-Gesellschaft (H.T.). J.S. and G.I. were supported by the Fondation pour la Recherche Médicale, P-Y.P was supported by grant from Région Ile-deFrance, Y.A. was supported by Deutscher Akademischer Austausch Dienst.

AUTHOR INFORMATION The authors declare no competing financial interests. Correspondence and requests for materials should be addressed to T.P. (thomas.preat@espci.fr) or H.T. (hiromut@neuro.mpg.de).

\section{AUTHOR CONTRIBUTIONS}

YA and HT designed and IS, SRT and VT carried out anatomical experiments. IS, YA and HT analyzed the microscopic data and assembled figures. KI and GMR provided Gal4 lines with their expression patterns. JS, GI, TP designed and JS, ST and PYP carried out behaviour experiments. JS carried out the P-replacement experiment. PYP, TP and PT designed in vivo imaging experiments and PYP carried out these experiments. JS, PYP, IS, YA, HT and TP wrote the paper. TP and HT supervised the work.

\section{Figure legends}


Figure $1 \mathrm{MB}-\mathrm{V} 2$ neurons connect the MB to the lateral horn and middle superiormedial protocerebrum.

Distribution of mCD8::GFP (white; a-c) and Syt::GFP (white; d-f) in the MB-V2 neurons driven by NP2492. Neuropils were labelled with an antibody against synapsin (orange). MB vertical lobes are outlined (dashed lines in $\mathbf{a}, \mathbf{d}, \mathbf{h}$ and $\mathbf{k}$ ).

a-c, A membrane reporter mCD8::GFP is localised in the terminals of MB-V2 neurons in the $\alpha$ - and $\alpha$ '-lobes of the MB (a), msmpr (b), and LH (c).

d-f, A presynaptic marker Syt::GFP is highly enriched in the terminals of MB-V2 neurons in the msmpr (e) and in the LH (f), but not in the MB vertical lobes (d).

g-I, Two subtypes of MB-V2 neurons - MB-V2a (g-i) and MB-V2 $\alpha^{\prime}-(\mathbf{j}-\mathbf{I})$ are identified by single-cell analyses. Schematic diagrams show the two types of MB-V2 neurons (blue) relative to the MB and LH (light green) $(\mathbf{g}, \mathbf{j})$. Dashed lines outline the brain surface. MB-V2 $\alpha$ neurons arborise in the MB $\alpha$ lobes (h) and project to the msmpr and the dorsal part of the LH $(\mathbf{i} ; n=87)$. MB-V2 $\alpha^{\prime}$ neurons arborise in the $\alpha^{\prime}$ lobes (k) and project to the msmpr, the ventral part of the $\mathrm{LH}$, and to a region ventromedial to the $\mathrm{LH}(\mathrm{I} ; n=44)$.

m-r, The polarity of the single MB-V2 $\alpha$ neuron visualized by a flp-out clone driving mCD8::GFP and Syt::HA using NP2492. The terminals of a single MB-V2a neuron in the $\mathrm{LH}(\mathbf{m}-\mathbf{o})$ and the MB and msmpr (p-r) are magnified.

Scale bars: $20 \mu \mathrm{m}$.

Figure 2 Output of the MB-V2 neurons is specifically required for the retrieval of short-lasting memory.

Temperature shift protocols are shown above each graph. 
a, Blocking MB-V2 neuron output during retrieval impairs 2-h memory. $\left(\mathbf{a}_{1}\right.$ : $F_{(2,39)}=$ 4.937, $p=0.0123$, ANOVA; $b_{2}: F_{(2,36)}=3.935, p=0.0185$, ANOVA). NP2492/UASshits and MZ160/UAS-shits are significantly different from their respective genetic controls. $n \geq 12$.

b, Blocking MB-V2 neuron output during training and consolidation does not affect 2h memory. ANOVA revealed no significant difference among the groups with $\left(\mathbf{a}_{1}\right)$ $N P 2492\left(F_{(2,32)}=1.337, p=0.28\right)$ and $\left(\mathbf{a}_{2}\right) \operatorname{MZ160}\left(\mathrm{F}_{(2,33)}=1.796, p=0.18\right) . n \geq 10$.

c, Expression of Shits in MB-V2 neurons does not affect 2-h memory when flies are conditioned and tested at permissive temperature. ANOVA revealed no significant difference among the groups with $\left(\mathbf{c}_{1}\right) \operatorname{NP2492}\left(\mathrm{F}_{(2,27)}=1.508, p=0.24\right)$ and $\left(\mathbf{c}_{2}\right)$ $\operatorname{MZ160}\left(\mathrm{F}_{(2,27)}=1.101, p=0.35\right) . n \geq 10$.

Each graph displays mean performance indices \pm SEM.

\section{Figure 3 Suppressing MB-V2 neurons in MZ160 rescues the memory defect}

a-c, Projection of the brain region including the MB lobes (light green).

In UAS-mCD8GFP; MZ160 (a), terminals of MB-V2 in the a lobe are visualized (arrows), whereas the signal is strongly reduced in combination with $C h a^{3.3 k b}-G A L 80$ (b) and NP2492-GAL80 (c). Scale bars: $20 \mu \mathrm{m}$.

d, Suppressing GAL4 expression in MB-V2 neurons with $C h a^{3.3 k b}-$ GAL80 restores a wild-type 2-h memory $\left(\mathrm{F}_{(4,45)}=3.305, p=0.0186\right.$, ANOVA). MZ160/UAS-shits differs significantly from UAS-shits, MZ160/+ and Cha ${ }^{3.3 k b}-G A L 80 /+; M Z 160 / U A S-s h i^{i s}$. Cha ${ }^{3.3 k b}-G A L 80 /+; M Z 160 / U A S-s h i^{\text {ts }}$ does not differ significantly from UAS-shits, MZ160/+ and Cha ${ }^{3.3 k b}-$ GAL80/+ ; +/UAS-shits $. n \geq 10$.

e, Suppressing GAL4 expression in MB-V2 neurons with NP2492-GAL80 restores a wild-type 2-h memory $(\mathrm{F}(2,36)=5.774, p=0.0067$, ANOVA). MZ160/UAS-shits differs 
significantly from NP2492-GAL80/+; MZ160/UAS-shits. NP2492-GAL80/+; MZ160/UAS-shis does not differ significantly from NP2492-GAL80/+; +/UAS-shits. Only females were used in this experiment. $n \geq 12$.

Each graph displays mean performance indices \pm SEM.

Figure 4 Output of the MB-V2 neurons is required for retrieval of consolidated long-lasting memories.

Temperature shift protocols are shown above each graph.

a, Blocking MB-V2 neuron output during retrieval impairs 24-h memory after massed conditioning $\left(\mathbf{a}_{1}: \mathrm{F}_{(2,39)}=4.822, p=0.0134\right.$, ANOVA; $\mathbf{a}_{2}: \mathrm{F}_{(2,27)}=12.95, p=0.0001$, ANOVA). NP2492/UAS-shit ${ }^{\text {ts }}$ and MZ160/UAS-shits are significantly different from their respective genetic controls. NP2492/UAS-shits and MZ160/UAS-shits do not differ significantly from 0 (one-sample T-test, $p=0.44$ and $p=0,72$ resp.). $n \geq 10$.

b, Blocking MB-V2 neuron output during retrieval impairs 24-h memory after spaced conditioning $\left(\mathbf{b}_{1}: \mathrm{F}_{(2,30)}=5.469, p=0.0094\right.$, ANOVA; $\mathbf{b}_{2}: \mathrm{F}_{(2,38)}=6.247, p=0.0037$, ANOVA). NP2492/UAS-shis $i^{t s}$ and MZ160/UAS-shits are significantly different from their respective genetic controls. NP2492/UAS-shits does not differ significantly from 0 (one-sample T-test, $p=0.25$ ). $n \geq 10$.

c, Blocking MB-V2 neuron output during spaced training and 2 hours after training does not affect 24-h memory. ANOVA revealed no significant difference among the groups with $\left(\mathbf{a}_{1}\right) \operatorname{NP2492}\left(\mathrm{F}_{(2,27)}=0.1157, p=0.89\right)$ and $\left(\mathrm{a}_{2}\right) \operatorname{MZ160}\left(\mathrm{F}_{(2,27)}=0.1936\right.$, $p=0.83) . n \geq 10$.

Each graph displays mean performance indices \pm SEM.

Figure 5 MB-V2 neurons are cholinergic. 
a-c, Cell bodies including the MB-V2 neurons in NP2492 do not overlap with the markers for glutamate, (DVGLUT; a), dopamine (TH; b) and GABA (GAD1; c). See also Fig. S6 for consistent results with $R 71 D 08$.

d-o, Terminals of the MB-V2 neurons in the LH and msmpr colocalize with ChAT (arrow), while the signal is occasionally undetectable in some terminals (arrowhead). The processes of MB-V2 is visualized with mCD8::GFP driven by R71D08. Terminals of a population of MB-V2 neurons (d-i) or a single MB-V2 a (j-o) are labelled with mCD8::GFP. See also Fig. S5 for consistent results with NP2492.

Scale bars: $10 \mu \mathrm{m}$.

Figure 6 MB-V2 neurons respond to olfactory stimuli in naïve flies.

a, Illustrative mean greyscale image of a time series acquisition showing a horizontal section of the right hemisphere of NP2492/+; UAS-GCaMP3/+ flies brain. MB-V2 neurons exhibited basal GCaMP3 fluorescence in their projections on the MB vertical lobes (i), msmpr (ii), lateral horn (iii) and in cell bodies (iv). The area delimited by dashed line includes $\mathrm{MB}$ and msmpr regions and was used to quantify odorant responses, here and in Fig. 7.

b-f, Colour coded variations of fluorescence in the same fly, after 2 s exposure to benzaldehyde (c), octanol (d), methylcyclohexanol (e) and isoamylacetate (f). MB-V2 neurons responded with calcium increase to all four odorants. Strong activation was observed in the MB and msmpr area, as delimited on (a). The calcium influx was much lower when no odorant was diluted in the paraffin oil (f).

g, Time course, averaged across all animals, of octanol (red; $\mathrm{n}=7$ ), methylcyclohexanol (blue; $n=7$ ) and oil (black; $n=5$ ) responses. The solid black bar indicates the delivery of the stimulus. The peak responses for all odorants, calculated 
as the temporal mean over the time window shaded in grey, are displayed in $\mathbf{h}$ (traces for benzaldehyde and isoamylacetate are not shown).

h, There were no significant differences among the mean responses to isoamylacetate $(\mathrm{I}, \mathrm{n}=3)$, benzaldehyde $(\mathrm{B}, \mathrm{n}=3)$, octanol (O) and methylcyclohexanol $(M)$, while the response to pure oil was significantly lower (oneway ANOVA, $\left.F_{(4,220)}=26.56, p<0.01\right)$.

\section{Figure 7 MB-V2 neurons show a reduced response to the trained odour after}

\section{conditioning.}

a, Overexpression of the GCaMP3 driven by NP2492-GAL4 did not significantly alter 3 hours memory performance ( $n=13$; one-way ANOVA, $\left.\mathrm{F}_{(2,36)}=2, p=0.15\right)$.

b, Sketch illustrating delivery of odours (black line) and electric shocks (red spikes) during the paired and unpaired training.

Items $\mathbf{c}, \mathbf{e}, \mathbf{g}, \mathbf{i}, \mathbf{k}$ and $\mathbf{d}, \mathbf{f}, \mathbf{h}, \mathbf{j}, \mathbf{l}$ display similar results of two sets of reciprocal experiments, performed either on flies trained with OCT (left) or MCH (right) as the CS+ odour ("CS+" in unpaired training). c-f: Mean greyscale images (scale bars: 15 $\mu \mathrm{m})$ of MB-V2 projections on msmpr, and colour coded patterns of the responses obtained to both odours in representative paired (c-d) and unpaired flies (e,-f). Paired flies specifically show a lower activation after CS+ than after CS- (arrowheads). Average time courses of odour responses are shown for paired (g-h) and unpaired (ij) flies. Black bars indicate the delivery of the stimulus. In paired flies, during the time window shaded in gray, the time course of CS+ response is decreased compared to the CS- response $\left(\mathbf{g} n=7, p=0.0035\right.$; $\left.\mathbf{h} n=6 p=4.5 \times 10^{-4}\right)$ while CS+ and CSresponses remain undistinguishable in unpaired flies (i $n=7 p=0.27 ; \mathbf{j} n=6 p=$ 0.13). The CS+ to CS- relative response, quantified as the natural logarithm of the 
$\mathrm{CS}+/ \mathrm{CS}-$ response, is lower in paired than in unpaired flies $(\mathbf{k}: p=0.0024 ; \mathbf{I}: p=$ $0.0043)$.

\section{Table 1 Sensory acuity controls.}

Response of naïve flies to the odorants used in the conditioning experiments at the restrictive temperature $\left(33^{\circ} \mathrm{C}\right)$. Temperature was shifted 30 min prior to the measurement of odorant avoidance. Flies have $1 \mathrm{~min}$ to choose between the aversive odour or air bubbled through mineral oil. For 3-octanol and 4-methycyclohexanol, one-way ANOVA revealed no significant differences between the genotypes (wild-type, UAS-shits, GAL4 and UAS$\left.s h i^{t s} / G A L 4 ; p>0.05\right) . n \geq 8$ groups. Each value displays mean performance indices $\pm \mathrm{SEM}$.

\section{Table 1}

Genotype Odour Avoidance

\begin{tabular}{lcc}
\hline & Octanol & Methylcyclohexanol \\
wild-type & $0.54 \pm 0.12$ & $0.53 \pm 0.10$ \\
UAS-shis & $0.51 \pm 0.08$ & $0.70 \pm 0.06$ \\
NP2492 & $0.45 \pm 0.10$ & $0.80 \pm 0.04$ \\
NP2492/+ UAS-shis $^{t s}+$ & $0.49 \pm 0.10$ & $0.78 \pm 0.07$ \\
\hline wild-type & $0.50 \pm 0.11$ & $0.56 \pm 0.10$ \\
UAS-shis & $0.49 \pm 0.08$ & $0.70 \pm 0.08$ \\
MZ160 & $0.59 \pm 0.09$ & $0.59 \pm 0.08$
\end{tabular}



$M Z 160 / U A S-s h i^{t s}$
$0.59 \pm 0.06$
$0.59 \pm 0.06$

\section{Methods}

Stocks. Flies were raised at $18^{\circ} \mathrm{C}, 60 \%$ humidity in a $12: 12$ h light:dark cycle. All strains were outcrossed to flies with a wild-type strain Canton-Special (Canton-S). The enhancer trap lines NP2492 [X] and MZ160 [III] were previously identified through a large-scale screen for mushroom body extrinsic neurons ${ }^{17,}{ }^{18}$. UAS-shits ${ }^{\text {ts }}$ [III] was used for behavioural experiments $^{19}$. The transformation vector for $R 71 D 08$ was generated using the $3.2 \mathrm{~kb}$ DNA fragment of the division abnormally delayed locus that was amplified with PCR and cloned into the transformation vector pBPGUw [27] (Primers sequence: GAAGTGCATGGCAAGGGAAGCAGAG and GCCAACCACTTATTTCTCGCCGTGT). For experiments with NP2492 whose insertion is on the X chromosome, only females of $N P 2492 /+; U A S-s h i^{t s} /+$ as well as the respective control genotypes were taken into account to calculate the memory index. NP2492-GAL80 line was obtained by crossing $y^{1}, w^{1118}$ females to $y^{l}, w^{1118}, N P 2492-G A L 4\left[w^{+}\right] ; \triangle H o p, C y O ; G A L 80\left[y^{+}\right], S b$ males. Positive events were detected by screening for $y^{1}, w^{1118}, G A L 80\left[y^{+}\right]$females on next generation and verified on confocal microscopy by crossing to NP2492-GAL4; UAS-mCD8::GFP.

Olfactory conditioning. For aversive training, flies were conditioned by an odour paired with electric shocks and subsequent exposure to a second odour in the absence of shock, as previously described ${ }^{39}$. Conditioning was performed on samples of 25-35 flies aged between 2 and 3 days with 3-octanol (>95\% purity; Fluka 74878, Sigma-Aldrich) and 4methylcyclohexanol (99\% purity; Fluka 66360, Sigma-Aldrich) at $0.360 \mathrm{mM}$ and $0.325 \mathrm{mM}$, 
respectively. Odours were diluted in paraffin oil (VWR international, Sigma-Aldrich). Memory tests were performed with a T-maze apparatus ${ }^{40}$. Flies could choose for $1 \mathrm{~min}$ between 2 arms delivering each a distinct odor. An index was calculated as the difference between the numbers of flies in each arm divided by the sum of flies in both arms. A performance index (PI) results from the average of two reciprocal experiments. For appetitive training, electric shocks were replaced by 1-minute sugar exposure, as previously described ${ }^{24}$.

Statistical analysis. We performed behavioural statistical analysis using GraphPad Prism 5.0 (GraphPad Software, Inc.). Each graph displays mean performance indices \pm SEM. One-way ANOVA was performed on each set of data followed by pairwise planned comparisons between relevant groups with a Student-Newman-Keuls test, except when specified. Wildtype flies were not taken in account for statistical analysis. For the data that violated the assumption of normal distribution (Supplementary Fig. 2j), non-parametric statistics was applied (Kruskal-Wallis test followed by Dunn's multiple comparisons). Asterisks denote significant differences with the post-hoc pair-wise comparisons $(* \mathrm{P}<0.05, * * \mathrm{P}<0.01, * * *$ $\mathrm{P}<0.001)$. NS: not significant.

Immunohistochemistry. We examined female F1 progenies (5-8 days after eclosion at $25^{\circ} \mathrm{C}$ ) between males of corresponding Gal4 lines and females carrying a single copy of UAS-mCD8::GFP [II $]^{22},-s y t:: G F P\left[\mathrm{III}{ }^{[20]},-s y t:: H A[\mathrm{X}],-n S y b:: G F P[\mathrm{III}],-m y r:: R F P[\mathrm{III}]\right.$ or their combination. For single-cell clonal analyses, $y w$ hsp70-flp; $U A S>C D 2 y$ >mCD8::GFP/CyO; TM2/TM6b ${ }^{[41]}$ females were crossed to NP2492, MZ160 or R71D08 males. One day post-eclosion, adult flies received a heat shock at $37^{\circ} \mathrm{C}$ for $30-45 \mathrm{~min}$ to remove the Flp-out cassette (CD2 y), and examined 5-7 days after heat shock. We analysed in total 131 single cell clones ( 87 for MB-V2 $\alpha$ and 44 for MB-V2 $\alpha$ ') with the three drivers. For 
single-cell analyses driving the membrane and presynaptic markers (i.e. mCD8::GFP and Syt::HA), UAS-mCD8::GFP UAS-Syt::HA hsp70-flp females were crossed to NP2492; $T u b>G A L 80>$ (II) males ${ }^{42}$. Late $3^{\text {rd }}$ instar larvae/early pupae of the progeny were heatshocked at $34^{\circ} \mathrm{C}$ for 20 minutes to remove the Flp-out cassette, and dissected after 10-12 days post-eclosion.

Brains were prepared by a standard immunolabeling procedure ${ }^{18,43}$, using antibodies against Drosophila GAD1 ${ }^{44,45}(1: 200), \operatorname{DVGLUT}^{46}$ (1:10.000), $\operatorname{ChAT}^{47}(1: 100), \mathrm{TH}^{48}(1: 1000)$, $\operatorname{Synapsin}^{49}$ (1:100), GFP (rabbit polyclonal, 1:1000, Invitrogen or rat monoclonal, 1:200, Chromotek), HA (1:200, Covance) and DsRed (1:100, Clontech). Coronal optical sections of whole-mount brains taken by confocal microscopy (Olympus FV1000) were analyzed with Image-J (NIH). In most figures, stained fibres and counterstaining were presented in white (RGB ratio; 255:255:255) and orange (255:85:0), respectively. Nomenclature of brain regions follows Otsuna and Ito $(2006)^{43}$.

\section{In vivo calcium imaging of odour responses}

Preparation of the flies: We targeted genetically the expression of the GCaMP3.0 calcium reporter to the MB-V2 neurons by combining a UAS-GCaMP3 [II] construct $^{29}$ with the NP2492-GAL4 driver. Measurements on naïve animals were performed on 1-2 days flies homozygous for both NP2492 and UAS-GCaMP3 transgenes. Female flies were caught without anaesthesia, then glued and operated as previously described for in vivo imaging ${ }^{50}$. The proboscis was also glued to the thorax to limit motion artefacts during image acquisition. The recording chamber was then placed beneath the water immersion $20 \mathrm{x}$ objective $(\mathrm{NA}=1$; Leica) of a TCS-SP5 laser-scanning confocal microscope (Leica), the brain of the fly being thus observed from the top. Experiments were performed at $20^{\circ} \mathrm{C}$, and the aperture on the top 
of the fly head bathed in a continuously flowing perfusion of Drosophila Ringer's solution (130 mM NaCl, $5 \mathrm{mM} \mathrm{KCl}, 2 \mathrm{mM} \mathrm{MgCl} 2,2 \mathrm{mM} \mathrm{CaCl}_{2}, 36 \mathrm{mM}$ sucrose, $5 \mathrm{mM}$ HEPES$\mathrm{NaOH} ; p H 7.3 ; 305$ mOsm) oxygenated by air bubbling.

For experiments on trained or unpaired flies, NP2492/+; UAS-GCaMP3/+ female flies with more moderate levels of reporter expression were used, in order to minimize physiological side effects of GCaMP3 expression. Consequently, GCaMP3-labelled areas and odourevoked activation patterns may slightly differ from experiments on naïve flies. One fly was captured $2 \mathrm{~h} 30$ after training and prepared with the same procedure, so that imaging actually took place approximately 3 hours after the end of the training protocol.

Odorant delivery: Each odorant was diluted 250 -fold $\mathrm{v} / \mathrm{v}$ in $100 \mathrm{~mL}$ paraffin oil in a glass bottle. Control bottles contained pure oil. A constant air stream, bubbling through pure oil, was directed at the fly throughout the recording. Following a trigger, a set of solenoid-driven valves redirected $20 \%$ of the air stream to the appropriate odour or control bottle for the aimed duration. The odour stream $\left(200 \mathrm{~mL} \cdot \mathrm{min}^{-1}\right)$ rejoined the primary stream $\left(800 \mathrm{~mL} \cdot \mathrm{min}^{-1}\right)$ $23 \mathrm{~cm}$ from the end of the delivery tube, which measured $4 \mathrm{~mm}$ in diameter. The end of the delivery tube was positioned $\sim 7-8 \mathrm{~mm}$ from the fly's antennae. All odorant concentrations are therefore $\sim 2000$-fold diluted $\left(5.3 \times 10^{-4} \mathrm{M}\right)$, not including the additional dilution factor between the end of the tube and the antennae.

Data acquisition and analysis: GCaMP3 probe was excited by the $488 \mathrm{~nm}$ line of an argon laser, scanning at a line rate of $400 \mathrm{~Hz}$. Fluorescence-induced light emission was collected by a photomultiplier in the 505-555 nm wavelength range. The pinhole was wide open, the collected light originated from thick horizontal sections of either of the two hemispheres of the brain.

Naïve flies were delivered each olfactory stimulus for $2 \mathrm{~s}$, and odorant presentations were spaced 3 min apart, in a random order. On each trained fly, a pair of responses to $1 \mathrm{~s}$ of CS+ 
and $\mathrm{CS}$ - odorants was recorded twice, to ensure that its olfactory ability was preserved throughout the experiment, but only the first presentation of each odour was kept for data analysis, to avoid desensitization effects. Within each pair, the odorants were pseudorandomly ordered. Overall, 12 flies received CS + first, and 14 flies received CS- first. During an experimental session, special care was taken to sequentially image, with minimal delay (55 min on average), on the same hemisphere and with the same odour delivered first, one paired and one unpaired fly grown in the same culture bottle, thus allowing treating paired and unpaired flies as paired data for statistical analysis. For this experiment, we focused on the msmpr as GCAMP signal was stronger in this region. All measurements were performed approximatively 3 hours after training.

Image analysis was performed offline with Matlab software using a custom-written program. To obtain the time course of the relative variation of fluorescence, the light intensity was averaged over a region of interest delimited by hand and surrounding the projections of the MB-V2 neurons in the area of the msmpr and mushroom bodies. The resulting time trace was normalized to a percent change of fluorescence $100 *\left(\mathrm{~F}-\mathrm{F}_{0}\right) / \mathrm{F}_{0}$, using a baseline value of the fluorescence $\mathrm{F}_{0}$, that was estimated as the temporal mean over the $1 \mathrm{~s}$ period preceding the switch of the valve. The peak response was calculated as the mean of this trace over a time window shown in gray on Fig. 6g and Fig. 7e,f. The same procedure was applied on a pixelby-pixel basis, yielding a colour coded image of the spatial pattern of the peak response. The brightness of each pixel was weighted by its mean fluorescence intensity over the duration of the whole acquisition, resulting in a shaded colour-coded image which highlights responses in regions that were actually labelled by the calcium reporter.

In the course of experiments on trained flies, comparison of responses to a given odorant was clouded by significant variability among different animals. To overcome this, a normalized value was calculated for each fly as the ratio of $\mathrm{CS}+$ over $\mathrm{CS}-$ peak responses. In order to 
accurately estimate the ratio of the mean responses, the natural logarithm of this value was averaged over all flies that had been submitted to the same training ${ }^{13}$. For statistical comparisons of two series of data, two-tailed t-tests were performed except when Lilliefors test indicated significant $(p<0.05)$ shift from normal distribution for any of the two series, in which case a Wilcoxon rank sum test was performed (Fig. 7j).

\section{References}

1. Keene, A.C. \& Waddell, S. Drosophila olfactory memory: single genes to complex neural circuits. Nat Rev Neurosci 8, 341-354 (2007).

2. McGuire, S.E., Deshazer, M. \& Davis, R.L. Thirty years of olfactory learning and memory research in Drosophila melanogaster. Prog Neurobiol 76, 328-347 (2005).

3. Tully, T., Preat, T., Boynton, S.C. \& Del Vecchio, M. Genetic dissection of consolidated memory in Drosophila. Cell 79, 35-47 (1994).

4. Masse, N.Y., Turner, G.C. \& Jefferis, G.S. Olfactory information processing in Drosophila. Curr Biol 19, R700-713 (2009).

5. Aso, Y., et al. Specific dopaminergic neurons for the formation of labile aversive memory. Curr Biol 20, 1445-1451 (2010).

6. Claridge-Chang, A., et al. Writing memories with light-addressable reinforcement circuitry. Cell 139, 405-415 (2009).

7. Schwaerzel, M., et al. Dopamine and octopamine differentiate between aversive and appetitive olfactory memories in Drosophila. J Neurosci 23, 10495-10502 (2003).

8. de Belle, J.S. \& Heisenberg, M. Associative odor learning in Drosophila abolished by chemical ablation of mushroom bodies. Science 263, 692-695 (1994).

9. Wang, Y., et al. Blockade of neurotransmission in Drosophila mushroom bodies impairs odor attraction, but not repulsion. Curr Biol 13, 1900-1904 (2003).

10. Crittenden, J.R., Skoulakis, E.M., Han, K.A., Kalderon, D. \& Davis, R.L. Tripartite mushroom body architecture revealed by antigenic markers. Learn Mem 5, 38-51 (1998).

11. Gervasi, N., Tchenio, P. \& Preat, T. PKA dynamics in Drosophila olfactory learning and memory center: coincidence detection by Rutabaga adenylyl cyclase and regulation by Dunce phosphodiesterase. Neuron 65, 516-529 (2010).

12. Tomchik, S.M. \& Davis, R.L. Dynamics of learning-related cAMP signaling and stimulus integration in the Drosophila olfactory pathway. Neuron 64, 510-521 (2009).

13. Wang, Y., Mamiya, A., Chiang, A.S. \& Zhong, Y. Imaging of an early memory trace in the Drosophila mushroom body. J Neurosci 28, 4368-4376 (2008).

14. $\mathrm{Yu}$, D., Akalal, D.B. \& Davis, R.L. Drosophila alpha/beta mushroom body neurons form a branch-specific, long-term cellular memory trace after spaced olfactory conditioning. Neuron 52, 845-855 (2006).

15. Isabel, G., Pascual, A. \& Preat, T. Exclusive consolidated memory phases in Drosophila. Science 304, 1024-1027 (2004).

16. McGuire, S.E., Le, P.T. \& Davis, R.L. The role of Drosophila mushroom body signaling in olfactory memory. Science 293, 1330-1333 (2001). 
17. Ito, K., et al. The organization of extrinsic neurons and their implications in the functional roles of the mushroom bodies in Drosophila melanogaster Meigen. Learn Mem 5, 52-77 (1998).

18. Tanaka, N.K., Tanimoto, H. \& Ito, K. Neuronal assemblies of the Drosophila mushroom body. J Comp Neurol 508, 711-755 (2008).

19. Kitamoto, T. Conditional modification of behavior in Drosophila by targeted expression of a temperature-sensitive shibire allele in defined neurons. J Neurobiol 47, 81-92 (2001).

20. Zhang, Y.Q., Rodesch, C.K. \& Broadie, K. Living synaptic vesicle marker: synaptotagmin-GFP. Genesis 34, 142-145 (2002).

21. Robinson, I.M., Ranjan, R. \& Schwarz, T.L. Synaptotagmins I and IV promote transmitter release independently of $\mathrm{Ca}(2+)$ binding in the $\mathrm{C}(2)$ A domain. Nature 418, 336340 (2002).

22. Lee, T. \& Luo, L. Mosaic analysis with a repressible cell marker for studies of gene function in neuronal morphogenesis. Neuron 22, 451-461 (1999).

23. Krashes, M.J. \& Waddell, S. Rapid consolidation to a radish and protein synthesisdependent long-term memory after single-session appetitive olfactory conditioning in Drosophila. J Neurosci 28, 3103-3113 (2008).

24. Colomb, J., Kaiser, L., Chabaud, M.A. \& Preat, T. Parametric and genetic analysis of Drosophila appetitive long-term memory and sugar motivation. Genes Brain Behav 8, 407415 (2009).

25. Kitamoto, T. Conditional disruption of synaptic transmission induces male-male courtship behavior in Drosophila. Proc Natl Acad Sci U S A 99, 13232-13237 (2002).

26. Sepp, K.J. \& Auld, V.J. Conversion of lacZ enhancer trap lines to GAL4 lines using targeted transposition in Drosophila melanogaster. Genetics 151, 1093-1101 (1999).

27. Pfeiffer, B.D., et al. Tools for neuroanatomy and neurogenetics in Drosophila. Proc Natl Acad Sci U S A 105, 9715-9720 (2008).

28. Yasuyama, K., Meinertzhagen, I.A. \& Schurmann, F.W. Synaptic organization of the mushroom body calyx in Drosophila melanogaster. J Comp Neurol 445, 211-226 (2002).

29. Tian, L., et al. Imaging neural activity in worms, flies and mice with improved GCaMP calcium indicators. Nat Methods 6, 875-881 (2009).

30. Mauelshagen, J. Neural correlates of olfactory learning paradigms in an identified neuron in the honeybee brain. J Neurophysiol 69, 609-625 (1993).

31. Okada, R., Rybak, J., Manz, G. \& Menzel, R. Learning-related plasticity in PE1 and other mushroom body-extrinsic neurons in the honeybee brain. $J$ Neurosci 27, 11736-11747 (2007).

32. Caporale, N. \& Dan, Y. Spike timing-dependent plasticity: a Hebbian learning rule. Annu Rev Neurosci 31, 25-46 (2008).

33. Bender, V.A., Bender, K.J., Brasier, D.J. \& Feldman, D.E. Two coincidence detectors for spike timing-dependent plasticity in somatosensory cortex. J Neurosci 26, 4166-4177 (2006).

34. Hashimotodani, Y., et al. Phospholipase Cbeta serves as a coincidence detector through its $\mathrm{Ca} 2+$ dependency for triggering retrograde endocannabinoid signal. Neuron $\mathbf{4 5}$, 257-268 (2005).

35. Menzel, R. \& Manz, G. Neural plasticity of mushroom body-extrinsic neurons in the honeybee brain. J Exp Biol 208, 4317-4332 (2005).

36. Liu, X. \& Davis, R.L. The GABAergic anterior paired lateral neuron suppresses and is suppressed by olfactory learning. Nat Neurosci 12, 53-59 (2009).

37. Bashir, Z.I. On long-term depression induced by activation of G-protein coupled receptors. Neurosci Res 45, 363-367 (2003). 
38. Tanaka, N.K., Awasaki, T., Shimada, T. \& Ito, K. Integration of chemosensory pathways in the Drosophila second-order olfactory centers. Curr Biol 14, 449-457 (2004).

39. Pascual, A. \& Preat, T. Localization of long-term memory within the Drosophila mushroom body. Science 294, 1115-1117 (2001).

40. Tully, T. \& Quinn, W.G. Classical conditioning and retention in normal and mutant Drosophila melanogaster. J Comp Physiol A 157, 263-277 (1985).

41. Wong, A.M., Wang, J.W. \& Axel, R. Spatial representation of the glomerular map in the Drosophila protocerebrum. Cell 109, 229-241 (2002).

42. Gordon, M.D. \& Scott, K. Motor control in a Drosophila taste circuit. Neuron 61, 373384 (2009).

43. Otsuna, H. \& Ito, K. Systematic analysis of the visual projection neurons of Drosophila melanogaster. I. Lobula-specific pathways. J Comp Neurol 497, 928-958 (2006).

44. Featherstone, D.E., et al. Presynaptic glutamic acid decarboxylase is required for induction of the postsynaptic receptor field at a glutamatergic synapse. Neuron 27, 71-84 (2000).

45. Sakai, T., Kasuya, J., Kitamoto, T. \& Aigaki, T. The Drosophila TRPA channel, Painless, regulates sexual receptivity in virgin females. Genes Brain Behav 8, 546-557 (2009).

46. Daniels, R.W., Gelfand, M.V., Collins, C.A. \& DiAntonio, A. Visualizing glutamatergic cell bodies and synapses in Drosophila larval and adult CNS. J Comp Neurol 508, 131-152 (2008).

47. Takagawa, K. \& Salvaterra, P. Analysis of choline acetyltransferase protein in temperature sensitive mutant flies using newly generated monoclonal antibody. Neurosci Res 24, 237-243 (1996).

48. Neckameyer, W.S., Woodrome, S., Holt, B. \& Mayer, A. Dopamine and senescence in Drosophila melanogaster. Neurobiol Aging 21, 145-152 (2000).

49. Klagges, B.R., et al. Invertebrate synapsins: a single gene codes for several isoforms in Drosophila. J Neurosci 16, 3154-3165 (1996).

50. Fiala, A. \& Spall, T. In vivo calcium imaging of brain activity in Drosophila by transgenic cameleon expression. Sci STKE 2003, PL6 (2003). 
Journal: Nature Neuroscience

\begin{tabular}{|l|l|}
\hline Article Title: & $\begin{array}{c}\text { Mushroom Body Efferent Neurons Responsible For Aversive Olfactory Memory } \\
\text { In Drosophila }\end{array}$ \\
\hline $\begin{array}{l}\text { Corresponding } \\
\text { Author: }\end{array}$ & Thomas Preat (thomas.preat@espci.fr) \\
\hline & \\
\hline
\end{tabular}

\begin{tabular}{|c|c|}
\hline $\begin{array}{l}\text { Supplementary } \\
\text { Item \& Number }\end{array}$ & Title or Caption \\
\hline Supplementary Figure 1 & $\begin{array}{l}\text { GAL4-expression patterns of NP2492 and MZ160 with } C h a^{3.3 k b}-G A L 80 \text { and } \\
\text { NP2492-GAL80 }\end{array}$ \\
\hline Supplementary Figure 2 & Polarity of MB-V2 neurons visualised with various reporters \\
\hline Supplementary Figure 3 & Other behavioural analysis of $N P 2492 / U A S-s h i^{t s}$ and $M Z 160 / U A S-s h i^{t s}$ flies \\
\hline Supplementary Figure 4 & MB-V2 are not glutamatergic, dopaminergic or GABAergic \\
\hline Supplementary Figure 5 & $\begin{array}{l}\text { Blockade of MB-V2 neurons using } R 71 D 08 \text { driver induces a 2-h memory } \\
\text { impairment }\end{array}$ \\
\hline Supplementary Figure 6 & Colocalization of MB-V2 terminals with ChAT \\
\hline Supplementary Figure 7 & Colocalization of MB-V2 terminals with ChAT but not DVGLUT in the LH \\
\hline Supplementary Figure 8 & No colocalization of MB-V2 terminals and DVGLUT in the LH and msmpr \\
\hline Supplementary Figure 9 & $\begin{array}{l}\text { No colocalization but proximity of MB-V2 terminals and GAD1 in the LH } \\
\text { and msmpr }\end{array}$ \\
\hline Supplementary Video 1 & Expression pattern of $N P 2492$ in the brain \\
\hline Supplementary Video 2 & Expression pattern of $M Z 160$ in the brain \\
\hline Supplementary Video 3 & Expression pattern of $M Z 160, C h a^{3.3 k b}-G A L 80$ in the brain \\
\hline Supplementary Video 4 & Expression pattern of $M Z 160, N P 2492-G A L 80$ in the brain \\
\hline Supplementary Video 5 & Expression pattern of $R 71 D 08$ in the brain \\
\hline
\end{tabular}



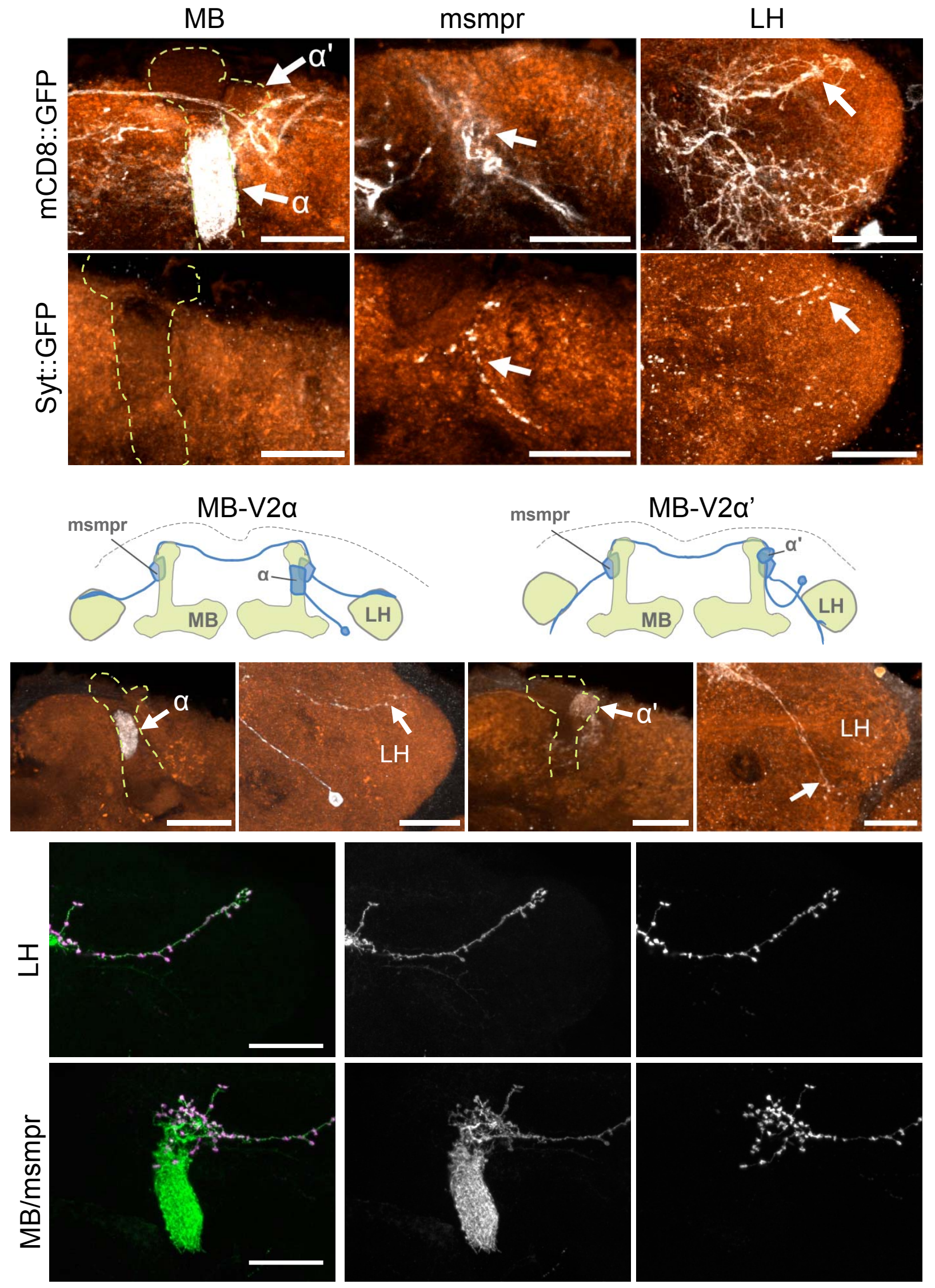

mCD8::GFP Syt::HA

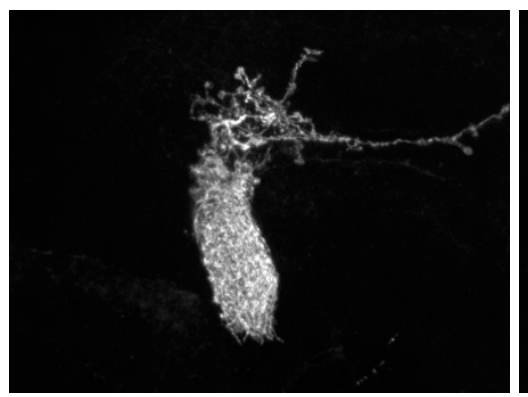

mCD8::GFP

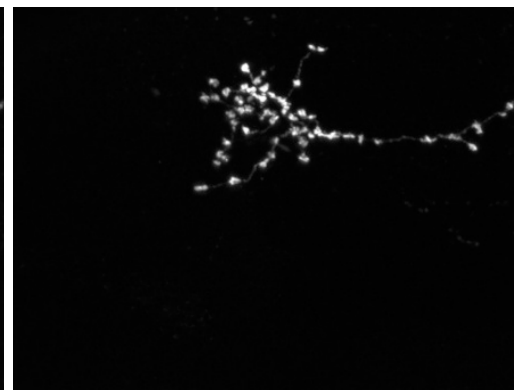

Syt::HA

Séjourné et al, Figure1 

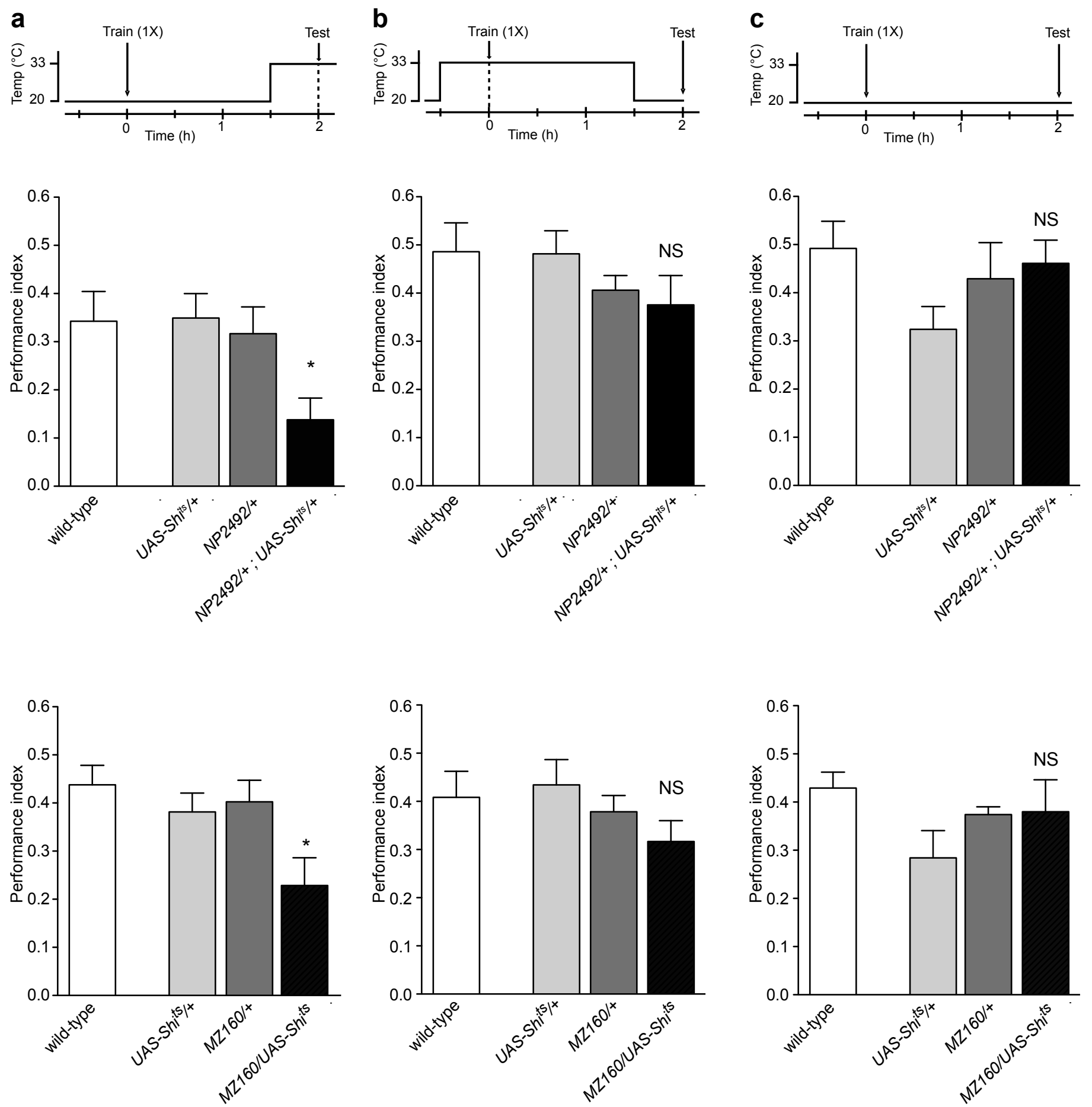

Séiourné ot al Fiaure? 

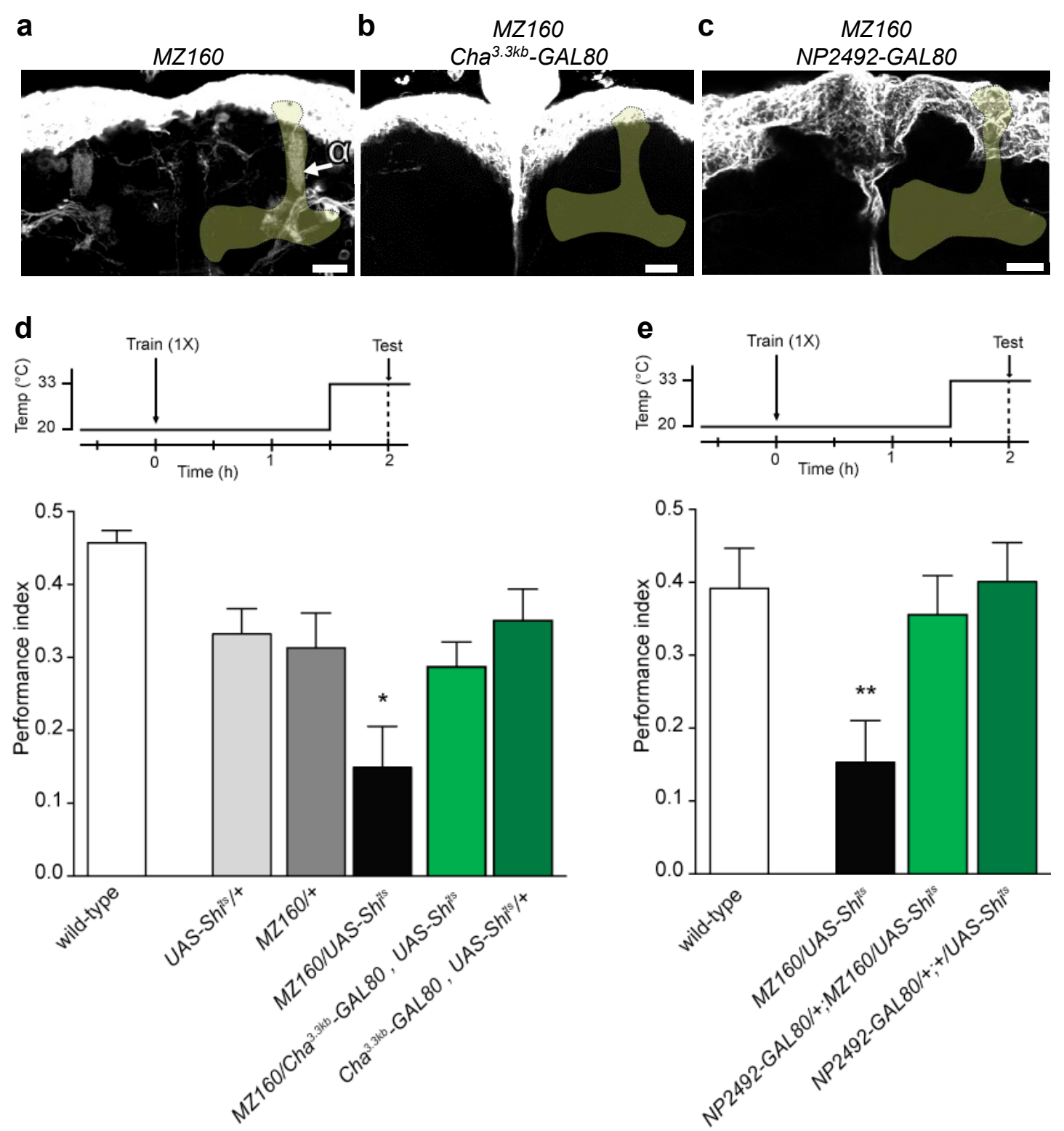
a
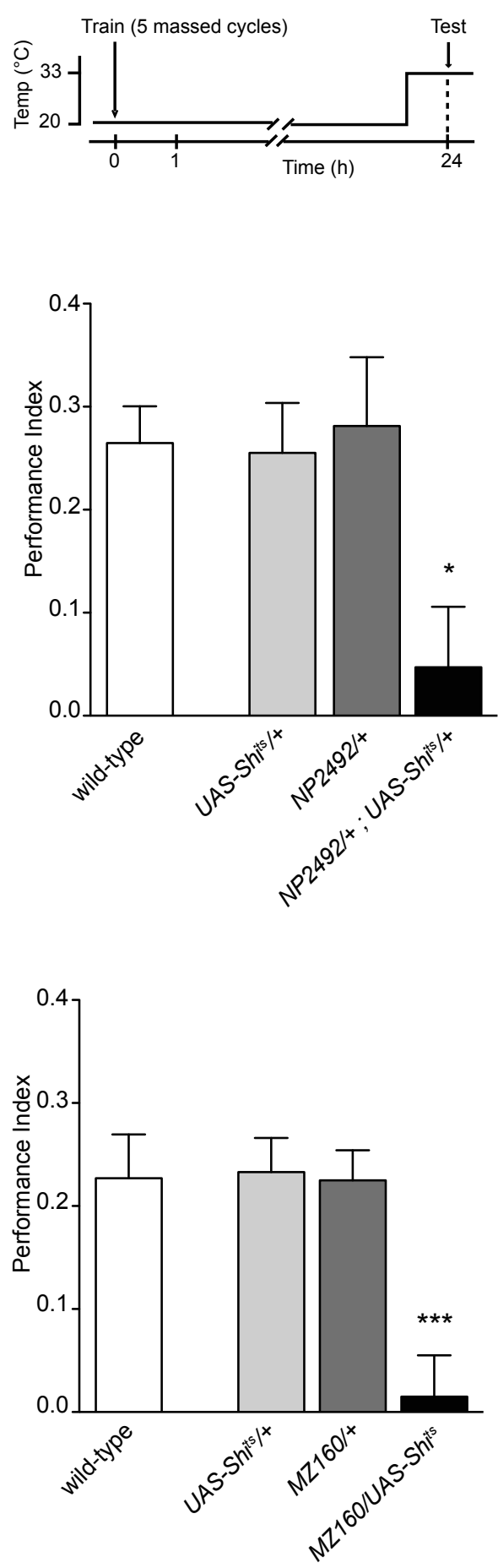

b
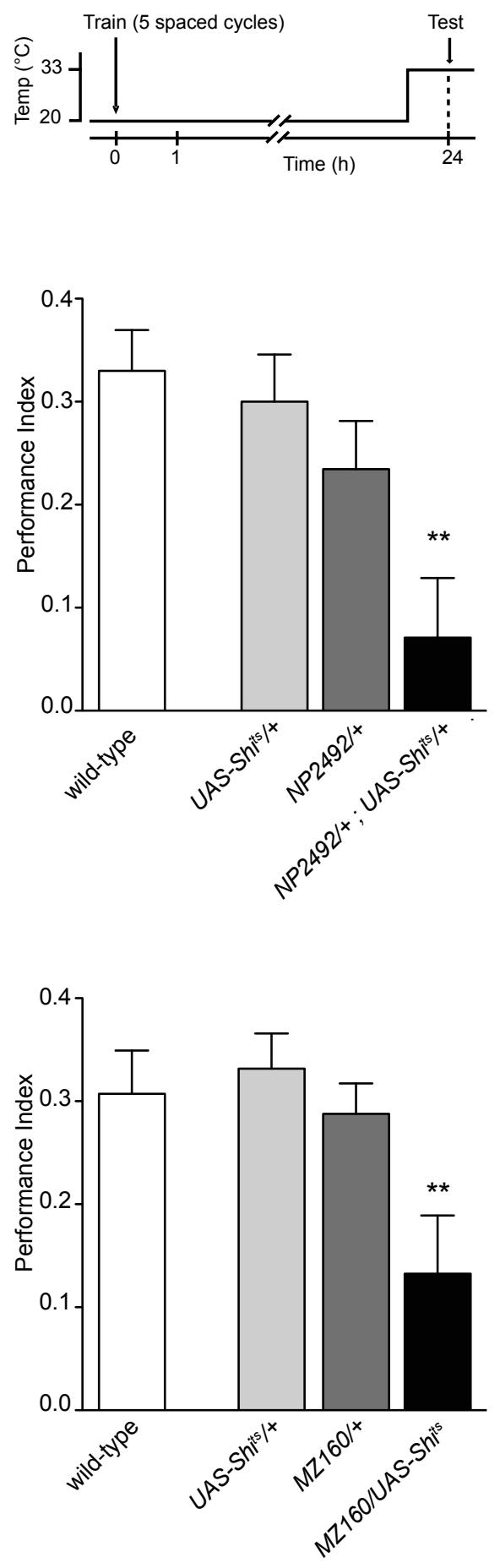

C
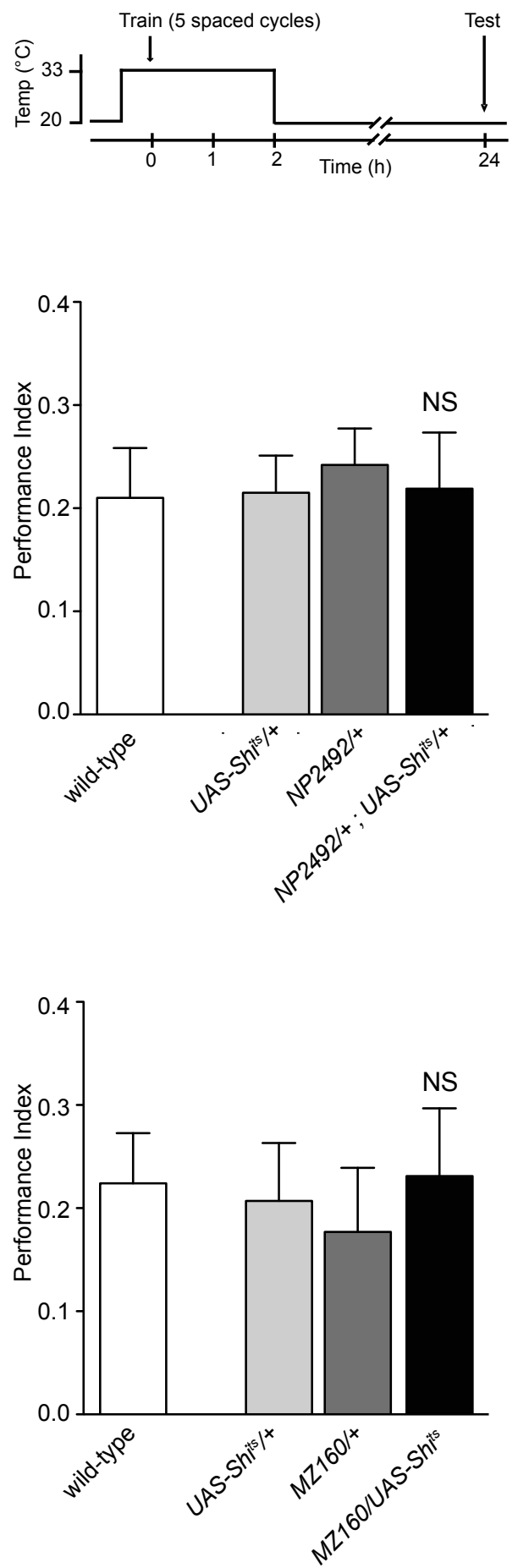


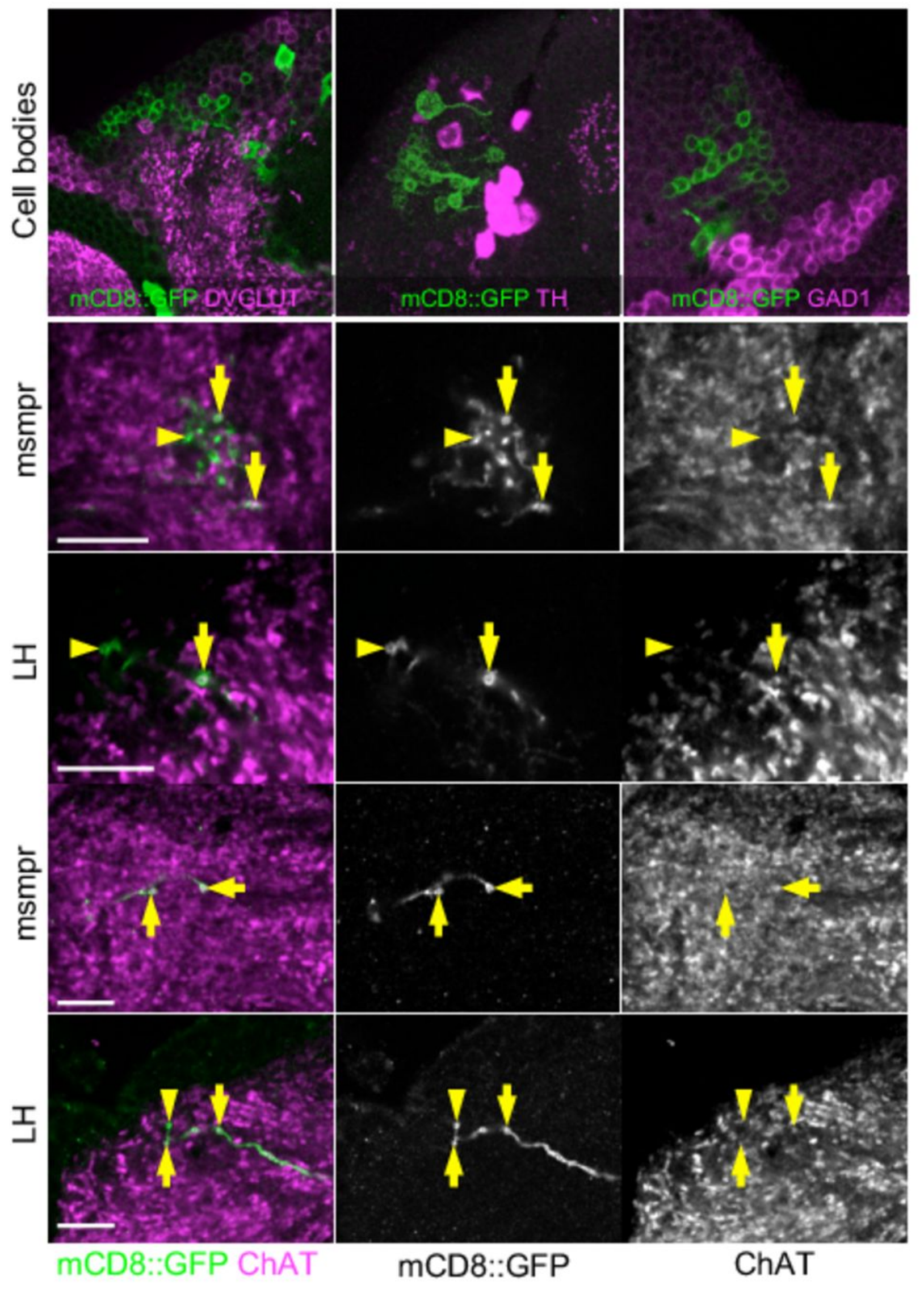

Fig. 5, Sejourne et al. 

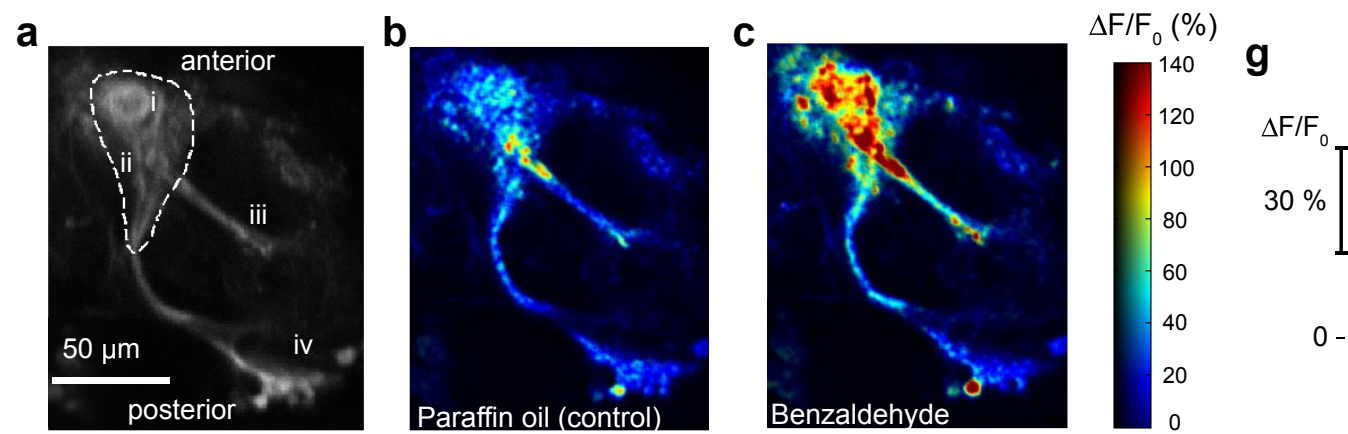

d
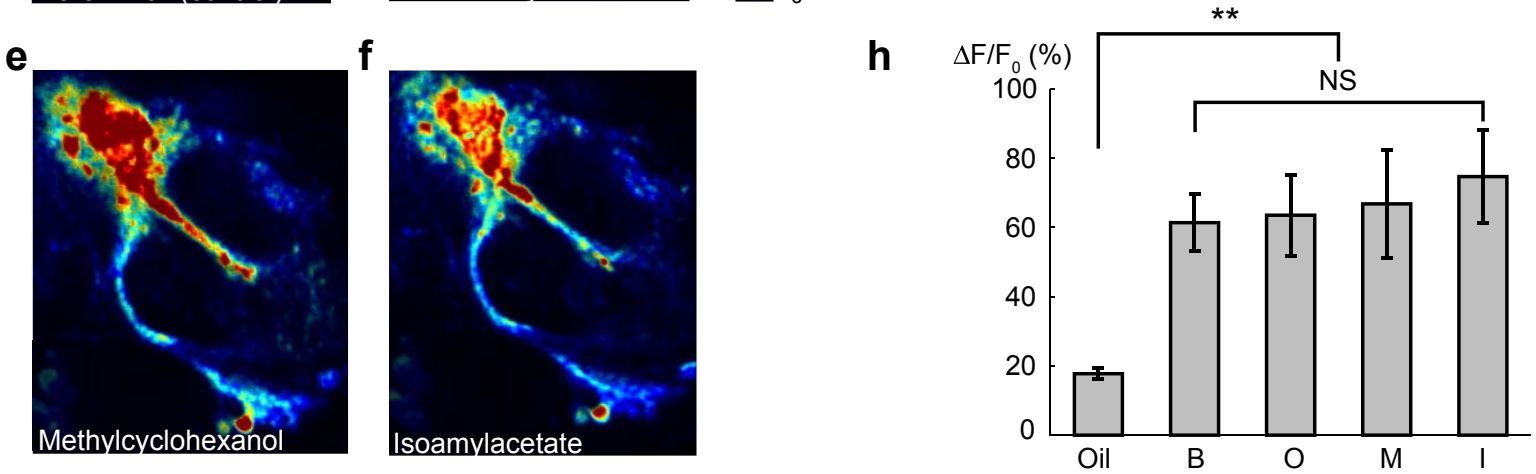

Séjourné et al., Figure 6 
a

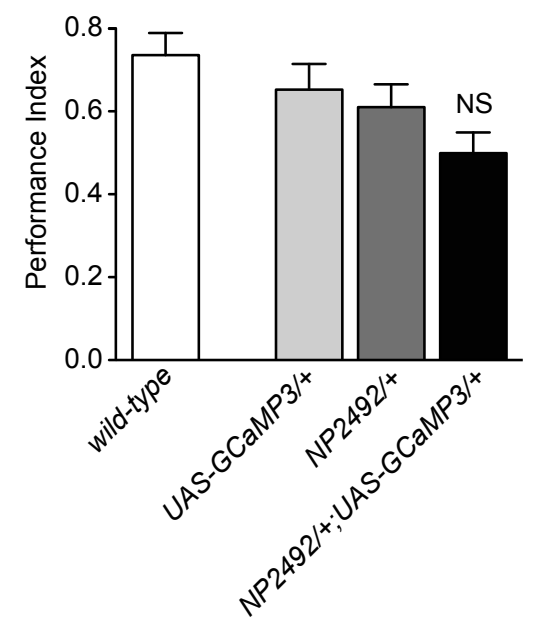

Octanol as CS+

C

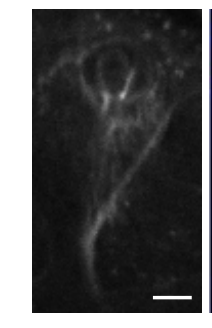

e

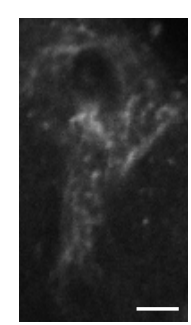

g

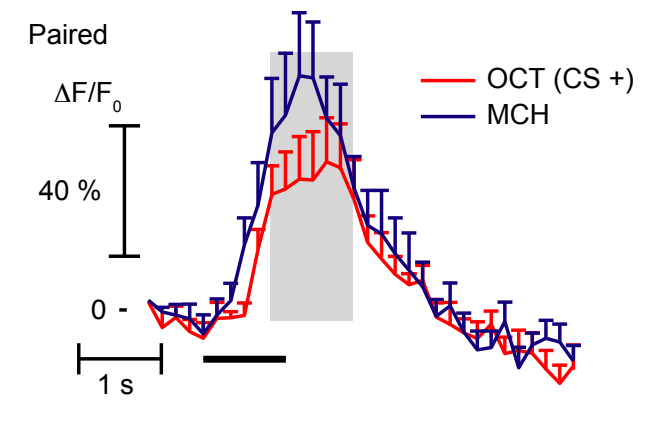

b

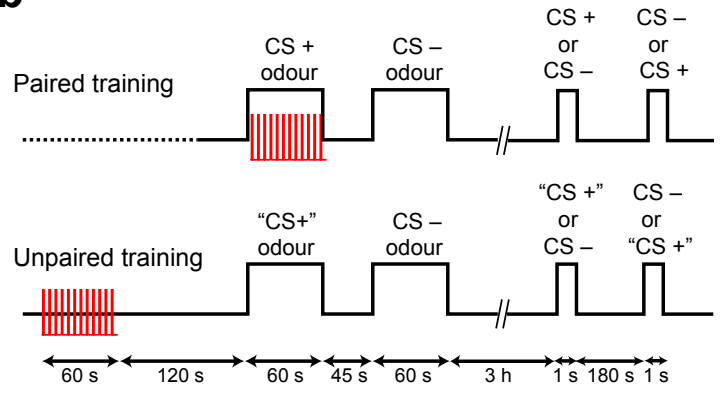

Methylcyclohexanol as CS+

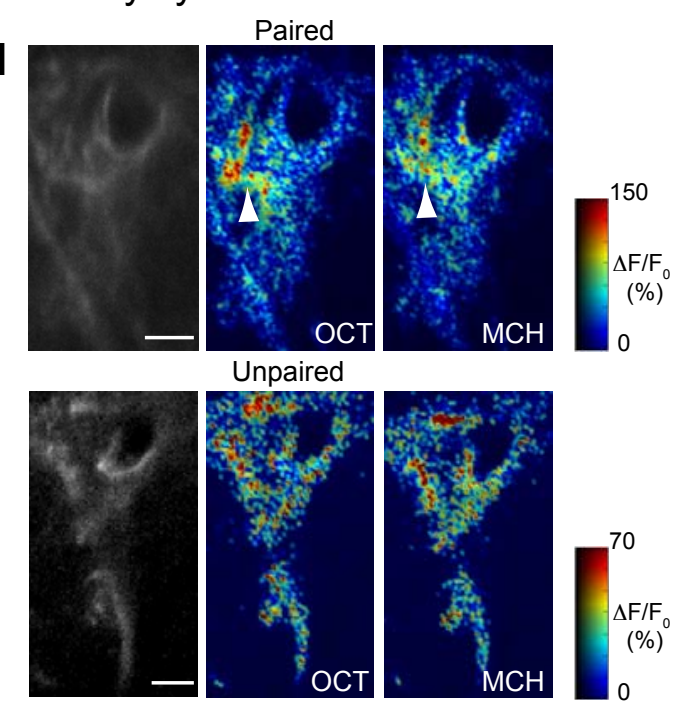

h

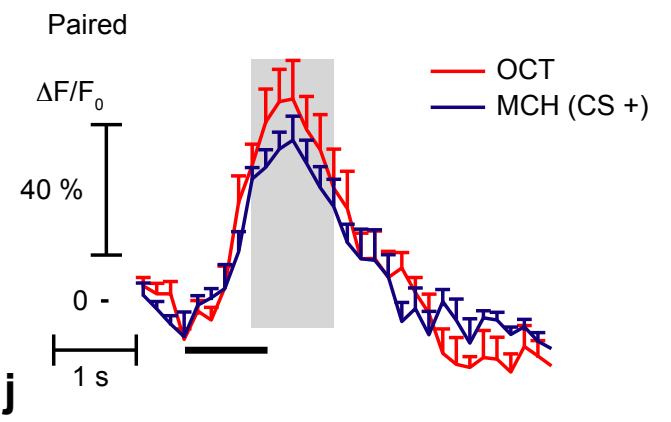

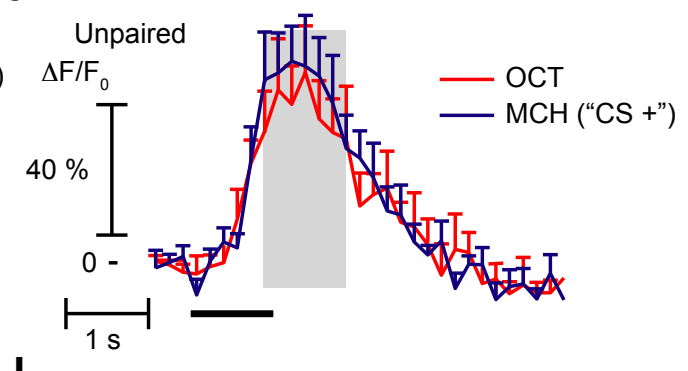

k
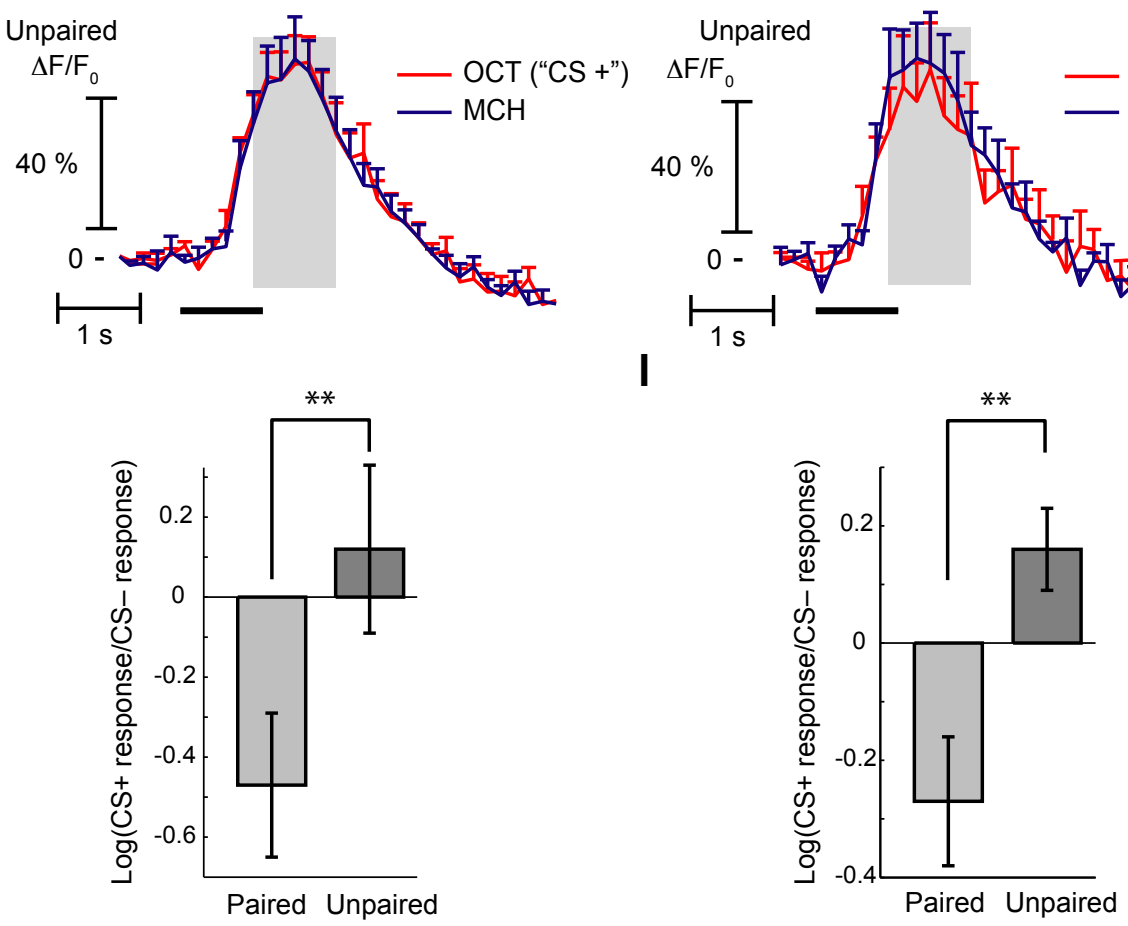

Séjourné et al., Figure 7 